\title{
Servicios de primaria y secundaria que comparten equipamiento. Experiencias en Gipuzkoa: posibilidades y dificultades
}

\author{
Jon Iribar Goenaga \\ Diputación Foral de Gipuzkoa \\ <jiribar@gipuzkoa.eus>
}

Gizarte Zerbitzuetako Euskal Legearen garapenak (2008) berarekin dakar azpiegitura fisiko bati loturiko zerbitzu berriak sortzea. Alderdi hori bereziki arazotsua gertatzen zaie horrelako eskumenak bete behar dituzten tamaina txiki eta ertainekoak diren udaletxeentzat, alde batetik inbertsioari loturiko gastu garrantzitsuak ageri direlako, eta bestetik, zerbitzu batzuen zeregina zentzudun bilakatzen delako zonalde edo eskualde zehatz zenbait udaletxeetara bideratzean, eta horrek zaildu egiten du azpiegitura sozial berrien sustapena. Horren ordezko aukera bat izan daiteke baliabide horiek sustatzea bigarren mailako arretako azpiegituretan batuz, dela jada abian daudenak, dela proiektaturik daudenak, eta bai titulartasun publikodunak bai pribatuak. Ordezko aukera horien esplorazioan lagungarri gertatzeko, artikulu honetan deskribatzen dira Gipuzkoan garatzen joan izan diren zenbait esperientzia, betiere helburu izanik berauen hedakuntza eta Euskadiko gizartezerbitzuen eremu osoari baliagarri gertatzea, baina berariaz udaletxe eta mankomunitateentzako.

\section{GAKO-HITZAK:}

Gizarte-zerbitzuak, udaletxeak, koordinazio administratiboa, araudia, eskumenak, esperientziak, Gipuzkoa.
El desarrollo de la Ley Vasca de Servicios Sociales (2008) implica la creación de nuevos servicios asociados a una infraestructura física. Esta cuestión resulta especialmente problemática para los ayuntamientos de tamaño pequeño o mediano que han de ejercer competencias de este tipo, en parte por los fuertes gastos que pueden estar asociados a la inversión, y en parte porque algunos servicios sólo tienen sentido si sirven a varios ayuntamientos de un área o comarca, lo que complica la promoción de nuevas infraestructuras sociales. Una alternativa es promover esos recursos asociándolos a infraestructuras de atención secundaria, tanto actuales como en proyecto, y tanto de titularidad pública como privada. Para contribuir a explorar esas alternativas, el presente artículo describe algunas experiencias que se han ido desarrollando en Gipuzkoa, con el objetivo de difundirlas y extraer algunas lecciones que puedan servir al conjunto de los servicios sociales de Euskadi, pero muy en particular a ayuntamientos y mancomunidades.

\section{Palabras Clave:}

Servicios sociales, ayuntamientos, coordinación administrativa, normativa, competencias, experiencias, Gipuzkoa. 


\section{Introducción}

El desarrollo de la Ley Vasca de Servicios Sociales (2008) implica, entre otras cosas, la creación de nuevos servicios asociados a una infraestructura física. Esta cuestión resulta especialmente problemática para los ayuntamientos de tamaño pequeño o mediano que han de ejercer competencias de este tipo, en parte por los fuertes gastos que pueden estar asociados a la inversión, y en parte porque algunos servicios sólo tienen sentido si sirven a varios ayuntamientos de un área o comarca, lo que exige acuerdos sobre ubicación, cofinanciación, prioridades de acceso y otras cuestiones que complican considerablemente la promoción de nuevas infraestructuras sociales.

Una alternativa que conviene explorar es la de promover esos recursos asociándolos a infraestructuras de atención secundaria, tanto actuales como en proyecto, y tanto de titularidad pública como privada. Para contribuir a explorar alternativas, el presente artículo describe algunas experiencias que se han ido desarrollando en Gipuzkoa, con el objetivo de difundirlas y extraer algunas lecciones que puedan servir al conjunto de los servicios sociales de Euskadi, pero muy en particular a ayuntamientos y mancomunidades.

La elección de Gipuzkoa se debe únicamente al limitado campo de conocimiento del autor, y de ninguna manera supone que estas experiencias no existan en Álava o Bizkaia, ni que las de Gipuzkoa sean especialmente modélicas. Finalmente, advertimos de que aquí no se aborda el asunto de la pura cesión de infraestructuras entre administraciones para el uso privativo de una de ellas, que es algo distinto de la coubicación o cogestión, y sobre la que existen, lógicamente, muchos más ejemplos; por ello, consideramos innecesario abundar en esa posibilidad.

\section{Experiencias}

\subsection{Servicios del Ayuntamiento de Donostia integrados en equipamientos residenciales de atención secundaria}

El Ayuntamiento de Donostia tiene servicios residenciales de competencia municipal destinados a personas mayores (apartamentos tutelados y viviendas comunitarias) integrados en una red propia y con una regulación común, pero mientras que algunos de estos equipamientos son de titularidad municipal, otros no lo son. Entre estos últimos podemos distinguir:

a. Servicios prestados en equipamientos forales que simultáneamente ofrecen servicios de atención secundaria: es el caso de los 18 apartamentos integrados en el centro residencial foral Txara II y de los 8 apartamentos integrados en el centro foral Elizaran (donde además hay un centro día de mayores y dos unidades de convivencia para personas con discapacidad y enfermedad mental, respectivamente). Esta fórmula se basa en convenios entre ambas administraciones.

b. Servicios contratados por el Ayuntamiento a centros que tienen como cliente principal a la Diputación Foral: es el caso de los 33 apartamentos para mayores integrados en el centro residencial Berra, o de los 17 apartamentos del mismo tipo ubicados en la Fundación Zorroaga. En este caso, la fórmula empleada es la contratación de servicios, sin ninguna intervención de la Diputación Foral.

\subsection{Servicios del Ayuntamiento de Tolosa integrados en un equipamiento residencial de atención secundaria}

En Tolosa, nos encontramos con un modelo singular desde el punto de vista formal o jurídico: el centro Uzturre es básicamente un equipamiento de atención secundaria, pero fue promovido por el Ayuntamiento en régimen de concesión de obra y gestión de servicios públicos, concesión a largo plazo que, en el ámbito de los servicios sociales, ha sido una fórmula empleada básicamente por las diputaciones forales y poco por los ayuntamientos.

Uzturre cuenta con 71 plazas residenciales para personas mayores dependientes y otras 40 de centro de día para ese mismo perfil, además de un módulo de 24 plazas destinado a personas con enfermedad mental; el cliente mayoritario de estos servicios es la Diputación Foral. Pero Uzturre cuenta además con 21 apartamentos, que suman 30 plazas; 15 de ellas están concertadas con el ayuntamiento, que las destina a personas mayores no dependientes o en riesgo de dependencia que están en riesgo de exclusión social.

\subsection{Servicios de otros ayuntamientos integrados en un equipamiento residencial de atención secundaria}

Se trata de apartamentos tutelados integrados en residencias de mayores cuya titularidad es municipal. Hernani y Andoain han hecho esta opción, como también la hizo Azkoitia (aunque, en este caso, ambos recursos han pasado a ser de titularidad foral).

\subsection{Servicios de la Diputación Foral integrados en un equipamiento residencial del Ayuntamiento de Zarautz}

El Ayuntamiento de Zarautz es titular de un edificio singular que ofrece 50 apartamentos tutelados, la mayoría destinados a personas mayores, pero con reserva de unos pocos para otras situaciones (piso de emergencia, acogida a víctimas de violencia machista), es decir, en su conjunto, para servicios residenciales de competencia municipal. La planta 
baja del edificio, sin embargo, acoge el centro de día para personas mayores en situación de dependencia, con 50 plazas, de competencia netamente foral.

La gestión de ambas partes del edificio es indirecta y adjudicada de modo integral por el Ayuntamiento, que a su vez concierta las plazas del centro de día con la Diputación. Además, y dado que existen apartamentos disponibles a la fecha, a partir del presente año la Diputación Foral va a utilizar tres de ellos para servicios de su competencia en el ámbito de la enfermedad mental. Ello supone tanto la cesión condicionada del Ayuntamiento como la incorporación de modificaciones al contrato de gestión indirecta actual.

\subsection{Servicios de la Diputación Foral integrados en un equipamiento residencial del Ayuntamiento de Zumaia}

El Ayuntamiento de Zumaia dispone de un equipamiento (el centro Branka) que agrupa tanto 16 apartamentos tutelados municipales como un centro de día de personas mayores con 20 plazas. La fórmula de gestión es similar a la empleada en Zarautz.

\subsection{Servicios de la Diputación Foral integrados en equipamientos municipales no residenciales}

Esta categoría de servicios engloba los centros de día para personas mayores de competencia foral que se encuentran integrados en centros del ayuntamiento que ofrecen otros servicios municipales, o incluso son la sede de servicios de otros sistemas públicos (como el centro de salud de Osakidetza, en el caso de municipios pequeños).

Históricamente, la tendencia más frecuente ha sido la de combinar el centro de día foral con el hogar del jubilado en el mismo edificio, en especial cuando el centro de día se crea por primera vez y su expectativa de 'clientes' es pequeña: con el tiempo y con el crecimiento de la demanda de centro de día, la tendencia posterior ha sido separar ambos servicios en diferentes ubicaciones. Podemos encontrar ejemplos de esta tendencia evolutiva en Zarautz, Azpeitia, Getaria, Errenteria y Eibar, entre otros municipios. El proceso está pendiente en localidades como Elgoibar o Bergara.

\subsection{Los centros rurales polivalentes}

En algunos municipios pequeños de Gipuzkoa, existen equipamientos bajo la forma de 'centros rurales polivalentes', promovidos en colaboración entre los respectivos ayuntamientos y la Diputación Foral. Su particularidad reside, por una parte, en la combinación de recursos de competencia municipal y foral no sólo integrados en un mismo edificio, sino gestionados de forma conjunta mediante un contrato de servicios suscrito por el ayuntamiento correspondiente; $y$, por otra, en una 'sobrefinanciación' por parte de la Diputación Foral, que pretende así compensar la falta de rentabilidad que supone, particularmente al inicio, la atención a pocas personas usuarias por parte de las empresas gestoras. Los equipamientos son siempre propiedad de los ayuntamientos o de las mancomunidades

Estos centros están situados en los siguientes municipios: Antzuola, Bidania-Goiatz, Elgeta, Errezil, Mendaro y Zegama. No todos ofrecen los mismos servicios ni están ubicados en equipamientos singulares, pero todos tienen en común el hecho de que están abiertos a una atención menos sectorizada según el reparto competencial (aunque las personas mayores dependientes sean los clientes principales, existen personas usuarias más jóvenes) y de que contemplan regímenes más flexibles de uso de los servicios (servicios concretos en días concretos, en vez de una estancia continuada y diaria), en comparación con servicios ubicados en centros urbanos.

\section{Instrumentos jurídicos y financieros para compartir equipamientos}

Los instrumentos básicos para materializar el uso compartido de equipamientos son los convenios entre administraciones cuando las infraestructuras son de titularidad pública. A este respecto, pueden distinguirse los convenios de carácter general de aquellos que tienen por objeto explícito la encomienda de gestión. Los primeros son los que utiliza la Diputación para concertar plazas, y suponen que el ayuntamiento, además de aportar la infraestructura, se encarga de la gestión (normalmente indirecta) del servicio. Los segundos sólo se dan hoy en día entre el Ayuntamiento de Donostia y la Diputación (casos de Txara II y Elizaran), y en ellos se establece explícitamente que el servicio 'instalado' en el edificio foral es de competencia municipal y se rige por la normativa municipal general para ese tipo de servicio (acceso, copago, reglamento), y no por la foral.

Esta distinción tiene su importancia: la encomienda de gestión, además de mantener el control regulatorio (municipal o foral) sobre el servicio, puede suponer que existan compromisos financieros adicionales al pago de una tarifa por estancia a la institución gestora (por ejemplo, inversiones o gastos de mantenimiento). 0 puede suponer una fórmula de pago distinta (una liquidación anual frente a un abono mensual, por ejemplo). A este respecto, cabe señalar que, en Gipuzkoa, aproximadamente la mitad de los centros de día para mayores (competencia foral) están en equipamientos municipales y son gestionados de modo indirecto por los ayuntamientos, sin que el instrumento jurídico recoja que se trata de una encomienda de gestión. Se trata de una situación en disonancia con el actual marco competencial que habrá que ir 
regularizando, bien a través de la encomienda o bien a través de la transferencia de la gestión al ente foral, manteniendo, en su caso, la cesión del local.

En cuanto a los servicios municipales integrados en recursos privados dedicados también a servicios de secundaria (como Berra-Donostia), el instrumento jurídico es el contrato entre las dos partes y la Diputación Foral no juega ningún papel: simplemente, el centro tiene dos clientes públicos en vez de uno.

\section{Lecciones extraídas}

De las experiencias señaladas, cabe extraer las siguientes lecciones:

- Las experiencias comentadas se dan en ambos sentidos: es decir, que es posible combinar recursos de atención primaria y secundaria tanto en centros forales como en municipales.

- También es posible compartir una infraestructura de titularidad privada.

- Casi todas estas experiencias se dan en servicios de atención a personas mayores (salvo algunos perfiles de usuarios en Uzturre-Tolosa y en los centros polivalentes), y no en servicios para otros colectivos.

- Las mayores dificultades de los ayuntamientos se refieren a la creación de estructuras residenciales de cierto tamaño (Zarautz es una excepción), y por ello resulta más eficiente acudir a la 'compra' de servicios. Sin embargo, a día de hoy existen muy pocas infraestructuras tanto forales como privadas que integren, por ejemplo, apartamentos tutelados, así que en la práctica la opción no es real. Por otra parte, la iniciativa privada no puede realizar esa oferta si no vislumbra una oportunidad de mercado (demanda pública solvente).

- El instrumento jurídico más acorde con el reparto competencial de los servicios sociales respecto al asunto que venimos tratando es la encomienda de gestión, que debería sustituir en algunos casos a los actuales convenios de colaboración, y sobre todo debería ser un elemento que habría que valorar en futuros desarrollos del Mapa de Servicios Sociales de Gipuzkoa. Ello es de particular importancia cuando se opta por una gestión unificada (es decir, por una única entidad gestora) para servicios de primaria y de secundaria.

- Las únicas experiencias en las que hay varios ayuntamientos implicados simultáneamente en este tipo de servicios son las correspondientes a las mancomunidades (centros rurales); en el resto de los casos, se trata de un único ayuntamiento que tiene algún tipo de acuerdo con la Diputación Foral. Como veremos a continuación, este modelo 'bilateral' presenta limitaciones importantes para el desarrollo de la atención primaria mediante nuevos servicios de centros.

\section{El desarrollo del Mapa de Servicios Sociales}

\subsection{Dificultades de desarrollo ligadas al Mapa y a la propia Ley de Servicios Sociales (2008)}

Un ejemplo muy claro de las dificultades para desplegar los objetivos establecidos por el mapa vasco o los mapas territoriales de servicios sociales es el diferente ámbito territorial de prestación de los servicios. Este asunto, que tiene mucho sentido al referirnos a una única institución que actúa en todo su territorio para cumplir los mandatos legales de acercamiento a los ciudadanos y de intervención lo más cercana posible a la comunidad, se convierte en un obstáculo muy grave cuando se intenta aplicar a muchas instituciones que, teóricamente, deben prestar servicios en espacios que desbordan los límites municipales, tanto territoriales como de población.

A su vez, esa dificultad está íntimamente conectada con el catálogo de la Ley de Servicios Sociales (2008), puesto que los servicios de competencia municipal que se establecen en ella no podrían ser los mismos si se hubieran definido como de obligada provisión en y por cada ayuntamiento. Por ejemplo, no tendría ningún sentido que todos y cada uno de los 251 ayuntamientos de Euskadi tuvieran que disponer, en su término municipal y exclusivamente a su costa, de viviendas tuteladas o de servicios de atención diurna para la exclusión social destinados sólo a sus vecinos.

El ámbito territorial de provisión y la necesidad de acción coordinada por los ayuntamientos no es, por tanto, algo que pueda modificarse simplemente mediante un cambio en el plan estratégico de los servicios sociales o en los mapas territoriales. Estos cambios pueden redefinir el número y alcance de los ámbitos más eficientes, pero siempre habrá al menos un nivel supramunicipal e infraterritorial para algunas de las competencias municipales vigentes. Por ello, el obstáculo se deriva directamente de la Ley, y no es tan sencillo ni cambiar la Ley ni sortear un obstáculo que la propia Ley ha creado. Entonces, ¿puede ser de ayuda el planteamiento que estamos examinando aquí? ¿La opción de compartir infraestructuras entre atención primaria y secundaria puede, en algunos casos, salvar el obstáculo?

\subsection{Posibilidades actuales}

Puesto que el único servicio municipal con infraestructura física que debe prestarse obligatoriamente en el propio municipio es el servicio social de base, y esto no plantea problemas especiales en la actualidad (más allá de las mejoras que deban hacerse), podemos distinguir tres situaciones para que los ayuntamientos pueden desarrollar más fácilmente sus 'nuevas' competencias sin crear recursos de la nada:

- Servicios en centros de día o de noche. 
- Apartamentos tutelados o viviendas comunitarias para mayores.

- Viviendas tuteladas para exclusión, pisos de acogida, pisos de emergencia.

\subsubsection{Servicios en centros de día o de noche}

Una posibilidad es utilizar servicios en centros de día o de noche que tienen como destinatarias a poblaciones parcialmente similares y que se distinguen por aspectos como la gama de prestaciones que se ofrecen, el horario y calendario de atención, la duración de la estancia o el perfil y ratio de profesionales estimado ${ }^{1}$, aunque sean competencialmente distintos. En definitiva, son los servicios que tienen nombre muy similar en el catálogo de la Ley (Cuadro 1).

\begin{tabular}{|c|c|c|c|}
\hline Primaria & $\begin{array}{l}\text { Servicio de } \\
\text { atención } \\
\text { diurna para } \\
\text { personas } \\
\text { mayores }\end{array}$ & $\begin{array}{l}\text { Servicio de } \\
\text { atención } \\
\text { diurna en } \\
\text { exclusión }\end{array}$ & $\begin{array}{l}\text { Servicio } \\
\text { de acogida } \\
\text { nocturna en } \\
\text { inclusión }\end{array}$ \\
\hline Secundaria & $\begin{array}{l}\text { Centro de día } \\
\text { para personas } \\
\text { mayores }\end{array}$ & $\begin{array}{l}\text { Centro de día } \\
\text { para atender } \\
\text { exclusión }\end{array}$ & $\begin{array}{l}\text { Centro de } \\
\text { acogida } \\
\text { nocturna en } \\
\text { inclusión }\end{array}$ \\
\hline
\end{tabular}

Fuente: Elaboración propia a partir del Decreto 185/2015.

Pueden existir sinergias al aprovechar equipamientos existentes o promover equipamientos nuevos que integren los dos niveles. En el caso de la atención diurna para mayores, se trata, en principio, de un 'programa', aunque pueda utilizar una o varias infraestructuras: una casa de cultura, un hogar del jubilado, un polideportivo o un centro de día. Por ejemplo, los municipios de Errenteria y Donostia se apoyan en los hogares del jubilado, de momento; y sabemos que el Ayuntamiento de Andoain está pensando en utilizar espacios del centro de día para este fin. En definitiva, es un servicio que debe implantarse, al menos inicialmente, a partir de lo que ya existe, sea municipal o foral.

Los servicios destinados a la exclusión/inclusión son un caso más claro todavía: primero, porque los de atención primaria están poco desarrollados o no existen en la mayor parte de Gipuzkoa, luego hay que promoverlos; segundo, porque son servicios de ámbito comarcal, según el Mapa; tercero, porque salvo los ayuntamientos grandes que ya disponen de algún servicio (e incluso alguno de éstos), es de esperar que los demás municipios propongan encomendar su gestión a la Diputación Foral. De hecho, en nuestra opinión, el desarrollo de estos servicios en la mayoría de las comarcas sólo está medianamente garantizado si se opta por

${ }^{1}$ Indicador no definido a día de hoy. una intervención de la Diputación, y ésta debería aprovechar para valorar la integración con servicios de su competencia.

\subsubsection{Apartamentos tutelados o viviendas comunitarias para mayores}

La de los apartamentos tutelados o viviendas comunitarias para mayores es la situación de más calado y con menos alternativas, porque el déficit es importante; porque no hay, de momento, una oferta privada que los ayuntamientos puedan concertar; porque la Diputación no tiene este tipo de recurso; y, especialmente, porque supone la promoción de equipamientos nuevos con un coste muy sustancial de inversión. Por poner un ejemplo, el Ayuntamiento de Oñati prevé construir un complejo de 16 apartamentos, con 20 plazas en total. El proyecto tiene un coste estimado de 1,8 millones de euros (IVA excluido), es decir, de más de 120.000 euros finales por cada apartamento, sin contar con el equipamiento ni el coste del suelo, que es público.

Parece, pues, evidente que merece la pena explorar alternativas más eficientes para ese coste, no tanto por la cuantía global, sino para maximizar los resultados: 16 apartamentos son pocos si tenemos en cuenta que estos servicios no tienen la misma rotación que las residencias, y que deben atender a situaciones de dependencia moderada que, en parte, se derivan actualmente a las residencias. Una forma de maximizar el resultado puede ser incorporar este recurso a nuevos servicios residenciales que promueva la Diputación Foral, y comentaremos más adelante las oportunidades concretas que se plantean en este momento.

Otra alternativa puede ser el recurso a la promoción general de viviendas de protección oficial, modificando proyectos actuales para que acojan este tipo de servicios (que no tienen por qué ser muy distintos de un edificio normal de viviendas). A este respecto, las residencias de Txara I y Txara II, en Donostia, fueron promovidas por el Gobierno Vasco en el marco de la creación de vivienda pública en el barrio de Intxaurrondo, y la Diputación Foral paga anualmente una cuantía durante cierto número de años, cuantía calculada según módulos de viviendas de protección oficial.

\subsubsection{Viviendas tuteladas para exclusión, pisos de acogida, pisos de emergencia}

Las viviendas tuteladas para exclusión, los pisos de acogida y los pisos de emergencia se parecen en que se trata de viviendas 'normales', y la diferencia entre ellos radica fundamentalmente en los servicios de apoyo que complementan el alojamiento. Según el Decreto de Cartera (2015), este apoyo pueden prestarlo los servicios sociales municipales, sin la dedicación de personal específico. Aunque nosotros ponemos en duda esta posibilidad (sobre 
todo, en exclusión social), a nuestros efectos lo relevante es que la infraestructura física no es 'especial' (viviendas hay en todos los municipios), lo que simplifica mucho la coordinación entre ayuntamientos o la necesidad de construir nuevos recursos.

En consecuencia, el margen de autonomía municipal es muy grande en estos servicios (especialmente si no va a dedicarse un personal especial): puede haber viviendas en varios ayuntamientos, incluso en todos los de una comarca; puede haber viviendas de un tipo en un municipio y de otro tipo en otro; pueden utilizarse viviendas de protección oficial, alquilar en el mercado inmobiliario, recurrir a EtxebideBizilagun o utilizar una vivienda en un complejo de apartamentos para mayores (recordemos el caso de Zarautz).

\section{Oportunidades}

Aludíamos hace un momento a la posibilidad de promover viviendas para mayores aprovechando proyectos sociales de envergadura de la Diputación Foral. Actualmente los proyectos que hay sobre la mesa y que se materializarán a medio plazo son los nuevos centros de Pasaia e Irun, que serán básicamente residencias de personas mayores con algunos servicios añadidos, todavía por concretar. Una característica común de esos centros es que sustituirán a las actuales residencias municipales en ambas localidades. Por lo tanto, se plantean dos posibles tipos de oportunidad:

a. Promover que los nuevos proyectos constructivos contemplen un módulo de apartamentos tutelados o vivienda comunitaria. Esto puede significar un menor coste en inversión para los ayuntamientos y un menor coste posterior de la gestión, así como facilitar el ámbito comarcal de cobertura.

b. Reconvertir los edificios de las residencias actuales en apartamentos o viviendas para servicios sociales de competencia municipal, una vez que existan los nuevos centros. Esto reforzaría el control del ayuntamiento sobre recursos de su competencia, pero supondría dedicar esfuerzos en solitario a la inversión y la posterior gestión, salvo que se llegara a un acuerdo de uso compartido con los ayuntamientos cercanos.

En ambas alternativas, deben posicionarse los ayuntamientos afectados, y preferiblemente del resto de la comarca. Para ello, la Diputación Foral debería abordar los proyectos citados con una visión global de necesidades y alternativas, contando con los ayuntamientos.

\section{Conclusiones}

En este artículo hemos visto que ya existen experiencias de infraestructuras compartidas entre primaria y secundaria, sin que ello signifique un criterio general o una planificación deliberada; simplemente, se han aprovechado oportunidades locales allí donde las había. También hemos visto que la mayoría de estas experiencias afectan a la atención a personas mayores y son más frecuentes cuando se trata de locales que de viviendas. Por lo tanto, existe una experiencia de gestión compartida o encomendada que puede ser útil para nuevos retos.

Hemos visto que el desarrollo del Mapa de Servicios Sociales tiene un obstáculo importante en la provisión de servicios de competencia municipal pero de ámbito supramunicipal, y que la intervención de la Diputación Foral puede ser decisiva, al menos en el ámbito de la exclusión/inclusión. En cambio, en el ámbito de las viviendas es donde los ayuntamientos van a tener que asumir un mayor protagonismo, si bien existen oportunidades concretas a medio plazo en algunas comarcas.

Finalmente, y de acuerdo con la autonomía municipal consagrada por la Ley de Instituciones Locales de Euskadi (2016), no es posible recomendar un modelo determinado de gestión a los ayuntamientos en general, pero es necesario plantear alternativas que desbloqueen el desarrollo del Mapa y hagan realidad el reparto competencial de la Ley de Servicios Sociales. 


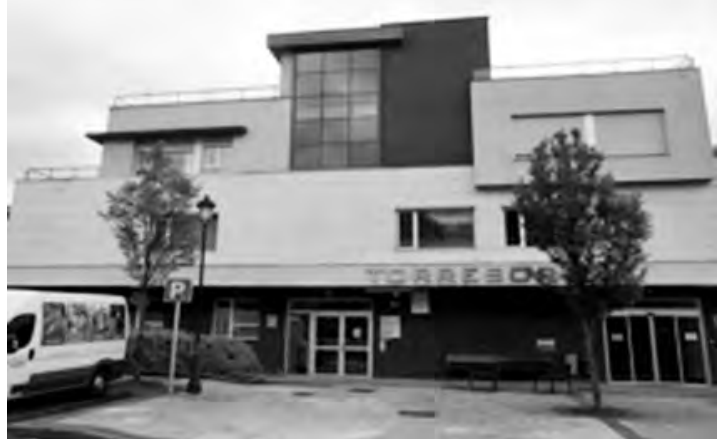

Centro Torresoroa (Antzuola).

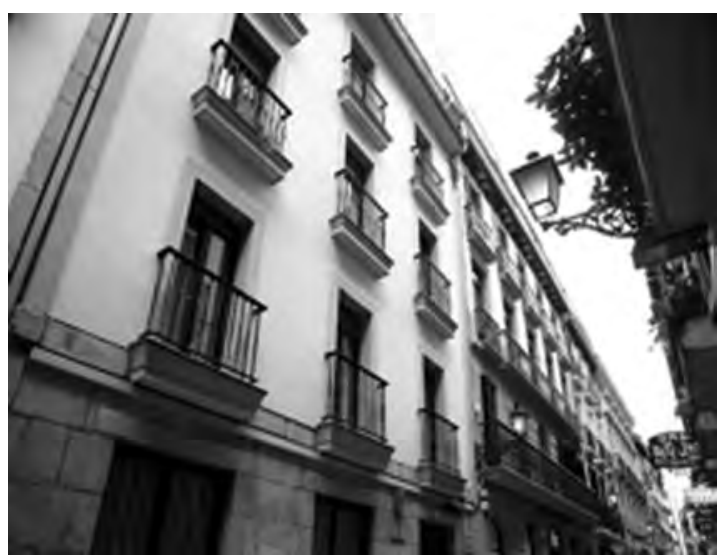

Centro Elizaran (Donostia).

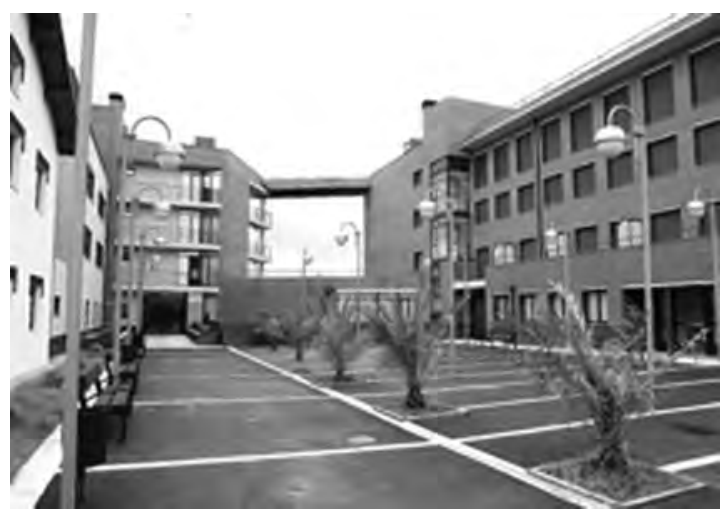

Centro Berra (Donostia).

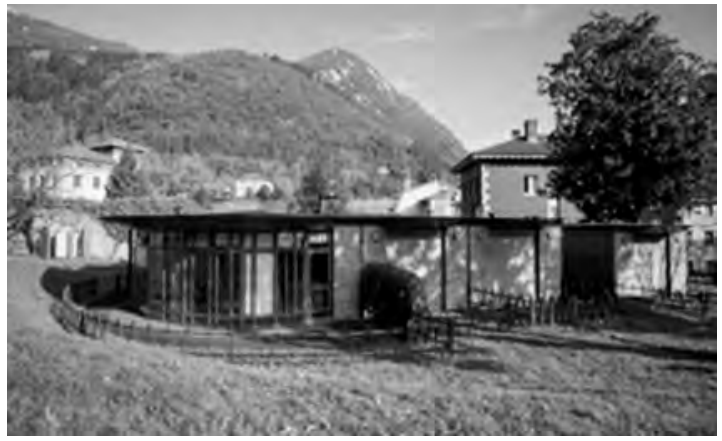

Centro rural polivalente (Mendaro).

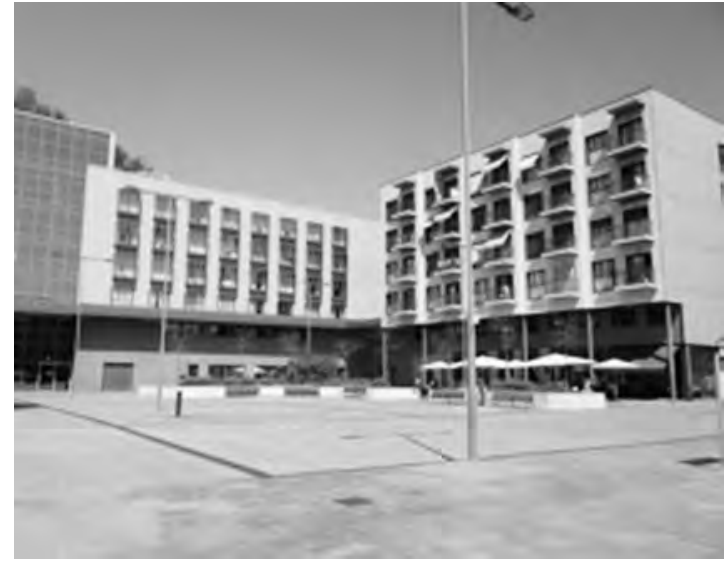

Centro Uzturre (Tolosa).



Centro Maria Txiki (Zarautz).

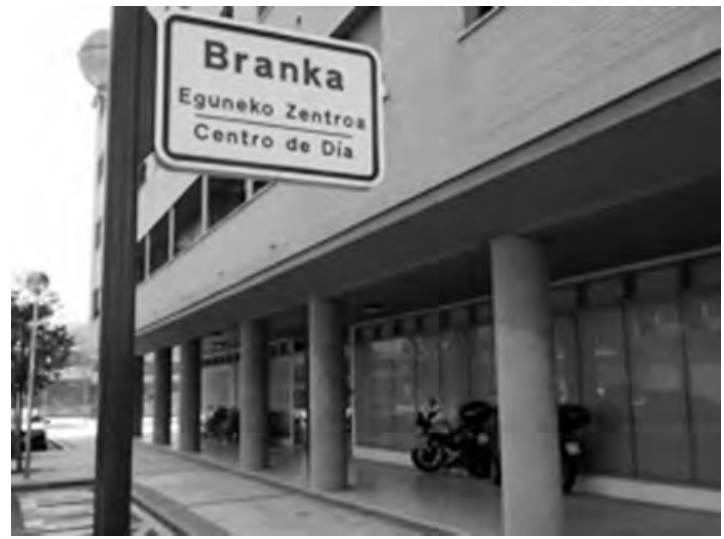

Centro Branka (Zumaia). 


\section{Legislación referenciada}

PAÍS VASCO (2016): “Ley 2/2016, de 7 de abril, de Instituciones Locales de Euskadi”, Boletín Oficial del País Vasco, no 70, 14-4-16 [rhttps:// www.euskadi.eus/y22-bopv/es/bopv2/ datos/2016/04/1601544a.shtmls].

- (2015): “Decreto 185/2015, de 6 de octubre, de Cartera de Prestaciones y Servicios del Sistema Vasco de Servicios Sociales", Boletín Oficial del País
Vasco, no 206, 29-10-15, págs. 1-87 [rhttps:// www.euskadi.eus/y22-bopv/es/bopv2/ datos/2015/10/1504561a.shtml〉].

- (2008): “Ley 12/2008, de 5 de diciembre, de Servicios Sociales", Boletín Oficial del País Vasco, no 246, 24-12-08, págs. 31.840-31.924 [<https://www.euskadi.eus/r47-bopvapps/es/ bopv2/datos/2008/12/0807143a.shtmlı]. 